\title{
Navigation of Mobile Robots Using WSN's RSSI Parameter and Potential Field Method
}

\author{
János Simon \\ Subotica Tech, Department of Informatics \\ Marka Oreškovića 16, 24000 Subotica, Serbia; e-mail: simon@vts.su.ac.rs
}

\section{Goran Martinović}

Faculty of Electrical Engineering, J. J. Strossmayer University of Osijek Kneza Trpimira 2b, 31000 Osijek, Croatia; e-mail: goran.martinovic@etfos.hr

\begin{abstract}
In the current work we present an algorithmic proposal for mobile robot navigation using a Wireless Sensor Network (WSN) for the location of a mobile measuring station in a controlled microclimatic environment. Another point of consideration is determining the navigation strategy. Publications in this field of robotics offer a large number of localization methods, mainly focusing on two fields: navigating locally and globally. Navigating locally will determine the mobile robot's position and orientation by implementing a series of sensors. Once we start from an initial position, the robot's position and orientation are updated continuously through the given time frame. Global navigation will ensure that the robot is able to determine its own position and orientation without having previously studied a map or being given some specific information.
\end{abstract}

Keywords - Localization;Sun SPOT; WSN; Mobile robot; RSSI

\section{Introduction}

A number of areas in engineering are centered on the problem of localization, so this field has been the focus of research for quite a long time. This is especially true for robotics, but the greatest challenge with indoor localization is posed by how effectively the GPS (Global Positioning System) can be used. This has been the reason for why WSNs (Wireless Sensor Networks) have been used more and more for the localization of a mobile object when indoors. [18] deals with the question of co-operative trilateration. The approach of the authors in that work used the exact range determination for its basis (implementing the ultrasound ranging technique as presented in [17]) for the solution of a least square problem on a large order system. The information needed for completely formulating the 
square problem requires the transmission from a possibly remote location through a number of hops. The study had a continuation in the form of the examinationof the scalability of the approach, as described in [19]. The authors of [21] were faced with the problem of how to localize in the absence of nodes. The role of the nodes is to create local coordinate systems and then continue to gather them to form a unique network coordinate system. Directional approaches are the topic of [20], which includes steerable directional antennas. [16] offers a presentation of how to estimate unknown node positions in a sensor network based solely on connectivity-induced constraints. The model of the known peer-to-peer communication within the network takes the form of a set of geometric constraints on the node positions. The global solution of a feasibility problem for these constraints results in estimated values for the unknown node positions within the network.This method [1] has several disadvantages: the central point of computation with the associated traffic overhead, scalability and reliability issues. The following section will give a more elaborate description of global navigation and the role it plays in this work.

\section{Global Navigation}

One of the central issues in numerous areas of engineering is the issue of global navigation when the indoor environment is not familiar. For robotics, exact location and orientation are of primary importance and have been the focus of studies for a number of years [7]. The key to successful indoor localization is whether or not the Global Positioning System can be used effectively. As a way of circumventing this challenge, the GPS is replaced by the Wireless Sensor Networks (WSNs) for helping with locating a mobile object in an indoor environment.

\subsection{Estimating Parameters}

This subsection will initially focus on the introduction of the model used for parameter estimation of the wireless sensor network. This paper presents the comparison of twoclassic propagation models. One of them is the least squares model, the other the maximum likelihood model, both used for the estimation of the parameters as it is fairly easy to influence the signal through noise and disturbance.The entire parameter estimation system has the following schematic structure:

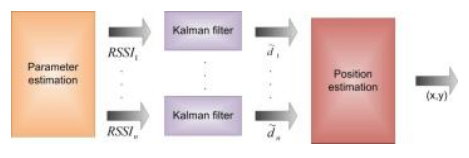

Figure 1

Parameter estimation 
As can be seen in this figure above, the extended Kalman filter is implemented, in order to relinearise every estimation once it has been calculated. The results in a new state estimate and the improved better reference state trajectory will subsequently be included in the estimation process, as described in [16].

\subsection{Algorithm for Triangulation}

A novel version of the Geometric Triangulation algorithm will be the focus of this part of the study, one whichworks withoutnode ordering and covers the whole navigation plane, with the possible exception ofseveral well-determined lines where localization is impossible [23]. Carefully defining the angles used by the algorithm is key to attaining improvements. The angles used are limited only by the common restrictions of all three object triangulation algorithms.

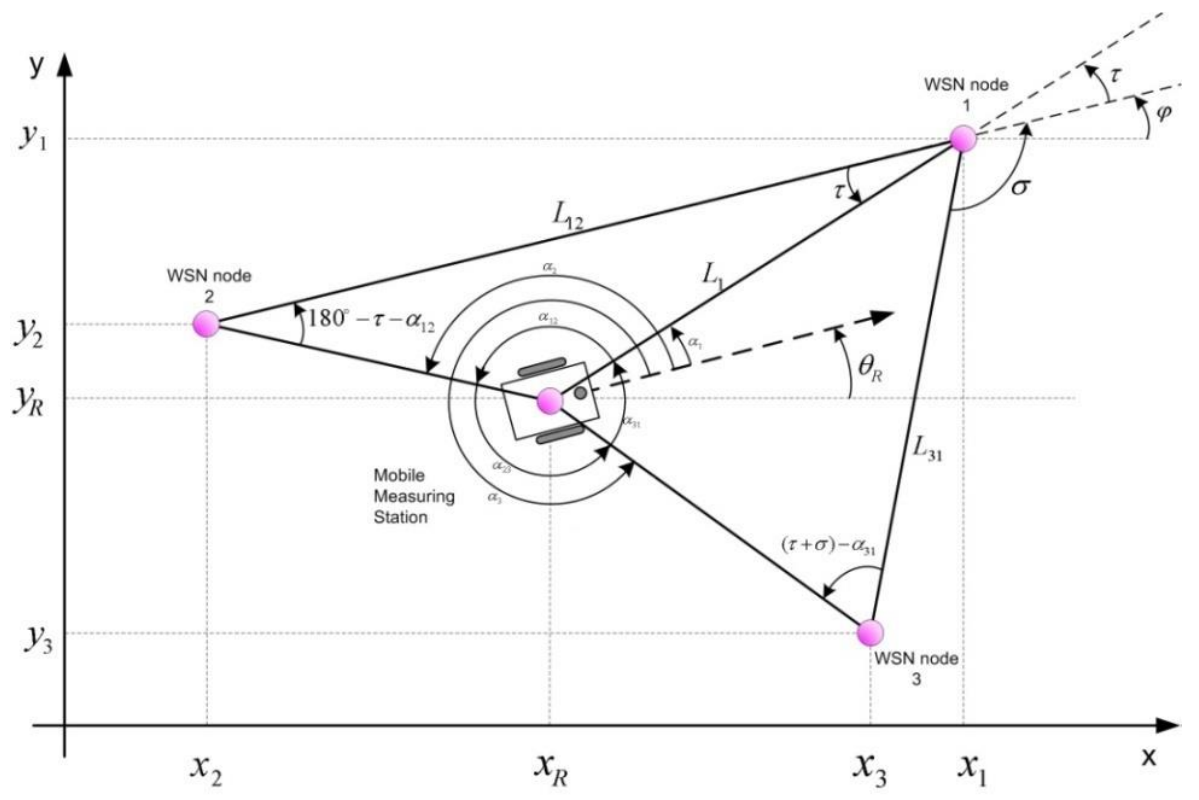

Figure 2

Geometric Triangulation algorithm

Let us take three distinguishable nodes in a Cartesian plane (Fig. 2); we will randomly label them 1,2 and 3 , and give them known positions $\left(\mathrm{x}_{1}, \mathrm{y}_{1}\right),\left(\mathrm{x}_{2}, \mathrm{y}_{2}\right)$ and $\left(\mathrm{x}_{3}, \mathrm{y}_{3}\right) . \mathrm{L}_{12}$ denotes the distance fromnode 1 to node $2 . \mathrm{L}_{31}$ will refer to the distance between nodes 1 and $3 . \mathrm{L}_{1}$ defines how far the robot is fromnode 1 . The aim is to determine the robot's position $\left(x_{R}, y_{R}\right)$ and orientation $\theta_{R}$. Thus, using the counterclockwise direction, the robot measures the angles $\alpha_{1}, \alpha_{2}$ and $\alpha_{3}$, denoting the orientation of the nodes in relation to the robot heading [23]. 
1. If there are less than three visible nodes available, then return a warning message and stop.

2. $\alpha_{12}=\alpha_{2}-\alpha_{1}$

3. If $\alpha_{1}>\alpha_{2}$ then $\alpha_{12}=360^{\circ}+\left(\alpha_{2}-\alpha_{1}\right)$

4. $\alpha_{31}=\alpha_{1}-\alpha_{3}$

5. If $\alpha_{3}>\alpha_{1}$ then $\alpha_{31}=360^{\circ}+\left(\alpha_{1}-\alpha_{3}\right)$

6. Compute $\mathrm{L}_{12}$ from known positions of WSN node 1 and 2 .

7. Compute $\mathrm{L}_{31}$ from known positions of WSN node 1 and 3.

8. Let $\varphi$ be an oriented angle such that $-180^{\circ}<\varphi \leq 180^{\circ}$. Its origin side is the image of the positive $\mathrm{x}$ semi-axis that results from the translation associated with the vector whose origin is $(0,0)$ and ends on node 1 . The extremity side is part of the straight line defined by nodes 1 and 2 whose origin is node 1 and does not go by node 2 .

9. Let $\sigma$ be an oriented angle such that $-180^{\circ}<\sigma \leq 180^{\circ}$. Its origin side is the straight line segment that joins nodes 1 and 3 . The extremity side is part of the straight line defined by nodes 1 and 2 whose origin is node 1 and does not go by node 2 .

10. $\gamma=\sigma-\alpha_{31}$

11. $\tau=\tan ^{-1}\left[\frac{\sin \alpha_{12}\left(L_{12} \sin \alpha_{31}-L_{31} \sin \gamma\right)}{L_{31} \sin \alpha_{12} \cos \gamma-L_{12} \cos \alpha_{12} \sin \alpha_{31}}\right]$

12. If $\left(\begin{array}{c}\alpha_{12}<180^{\circ} \\ \tau<0^{\circ}\end{array} \quad\right.$ then $\quad \tau=\tau+180^{\circ}$

13. If $\left(\begin{array}{c}\alpha_{12}>180^{\circ} \\ \tau>0^{\circ}\end{array} \quad\right.$ then $\quad \tau=\tau-180^{\circ}$

14. If $\left|\sin \alpha_{12}\right|>\left|\sin \alpha_{31}\right| \quad$ then $\quad L_{1}=\frac{L_{12} \sin \left(\tau+\alpha_{12}\right)}{\sin \alpha_{12}}$

15.

else $\quad L_{1}=\frac{L_{31} \sin \left(\tau+\sigma+\alpha_{31}\right)}{\sin \alpha_{31}}$

16. $x_{R}=x_{1}-L_{1} \cos (\varphi+\tau)$

17. $y_{R}=y_{1}-L_{1} \sin (\varphi+\tau)$

18. $\theta_{R}=\varphi+\tau-\alpha_{1}$

19. If $\theta_{R} \leq-180^{\circ}$ then $\theta_{R}=\theta_{R}+360^{\circ}$

20. If $\theta_{R} \geq 180^{\circ}$ then $\theta_{R}=\theta_{R}-360^{\circ}$

Figure 3

Geometric Triangulation algorithm 
Fig. 3 presents the algorithmic approach, which is capable of yielding considerable accuracy [23]. In terms of how costly an approach is, the RSS is a rather cheap way of using techniques that measure the power of the received signal strength, which will then transform the measured RSS value into a distance. The RSS method has the added advantage that it does not need any furthermeasuring hardware.

\subsection{Measuring and Estimating Parameter Using RSSI}

In order to measure the RSSI, a chip with the specification of CC2440 was used. The characteristics include an onboard antenna, and the chip operates within the 2.4 GHz ISM band. This device comprises the node's "heart" used in the current research $[11,12]$. The chip supports the IEEE 802.15.4/ZigBee protocol. The relationship between measured RSSI values and distances is shown in the figure below $[4,10]$.

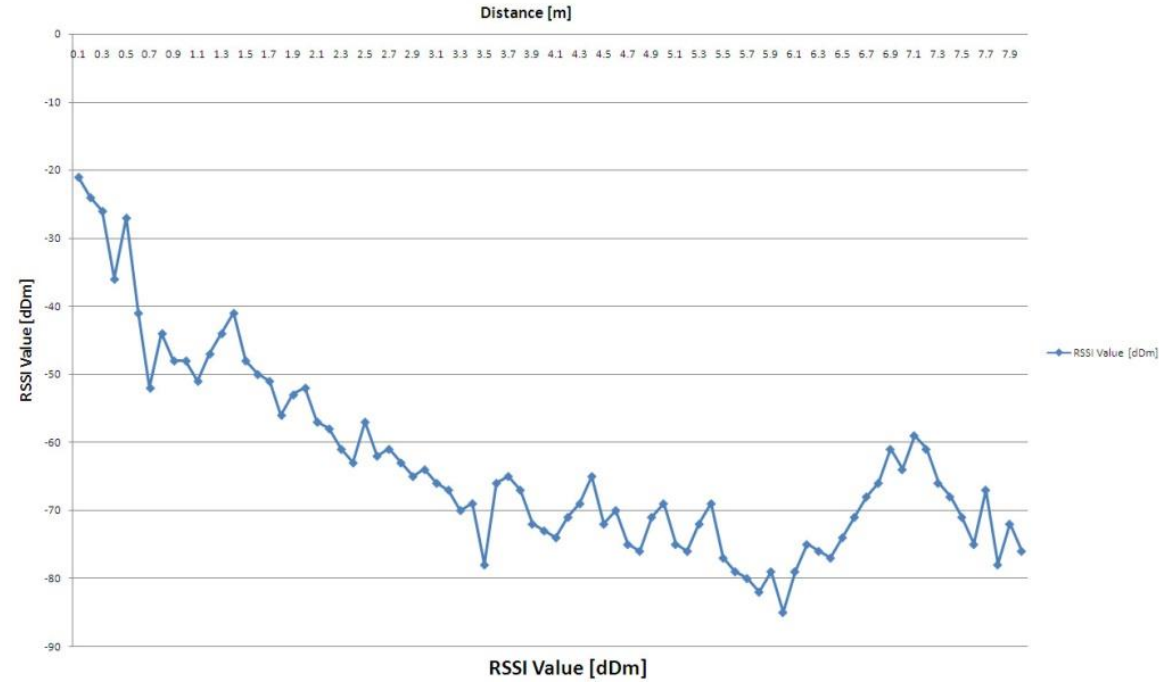

Figure 4

Measured RSSI values

Fig. 4 presents the following: the curve showing the relationship between the RSSI values and the distance is not a smooth one. Due to the multi-path interference, the measured values vary randomly [22, 9]. So the keystone of this model was the rationalization of parameters. 


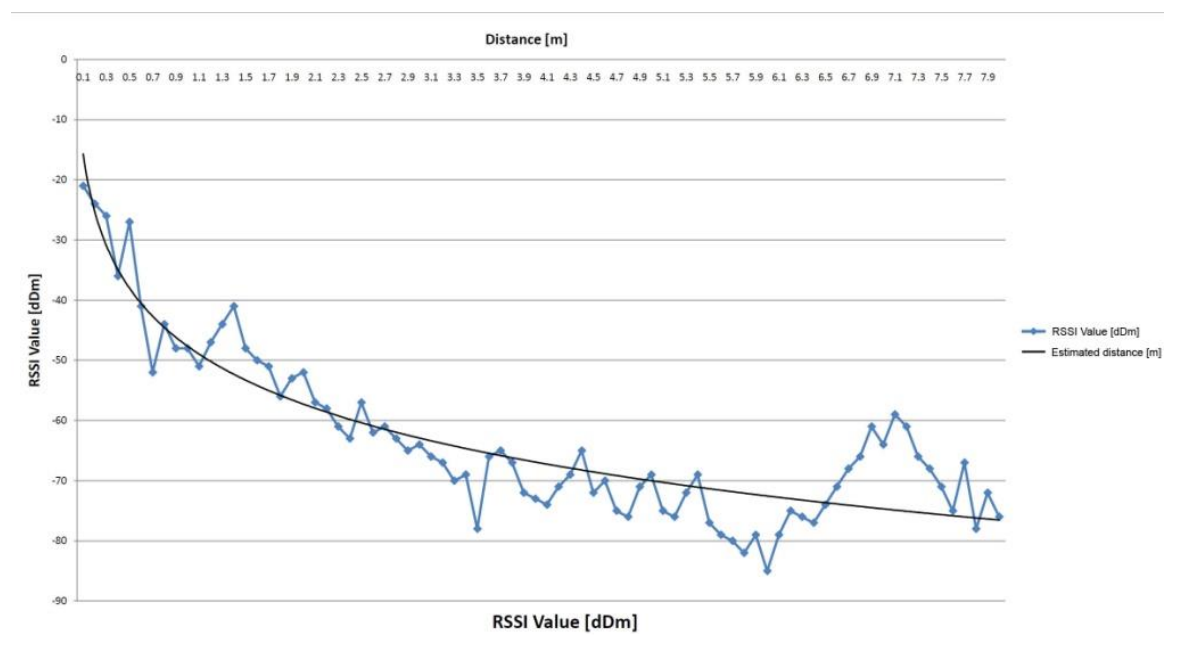

Figure 5

Estimated distance based on measured RSSI

\section{Local Navigation}

For path planning and collision avoidance, the Potential Fields Approach [15,8] is one of the most often used options, mainly due to how simple and elegant it is in a mathematical sense. It is simple to implement and yields respectable and rapid results for real-time navigation without delay [14]. The objective of any path planning algorithm is to flee from obstacles and move towards a desired goal. The basic idea behind this method is the idea of attractive and repulsive forces. In potential fields, the aim would be defined as a global minimum potential value, with all obstacles seen as high valued potential fields. How the robot moves will be defined by the attained values which appear in its path; in an ideal situation the values will shift from high to low potentials. There are two major formulations, the Local Potential Approachand the Global Potential Approach. In this work we consider only the Local Potential Approach. For this approach, there are several different types of potential field functions, differing from each other by the way the potential is calculated.

\subsection{Repulsive Potential Field}

The repulsive potential field is the element that keeps the robot away from the obstacles encountered on its path. Every repulsive action vector points away from the obstacle surface, directing the robot to bypass the obstacle. 


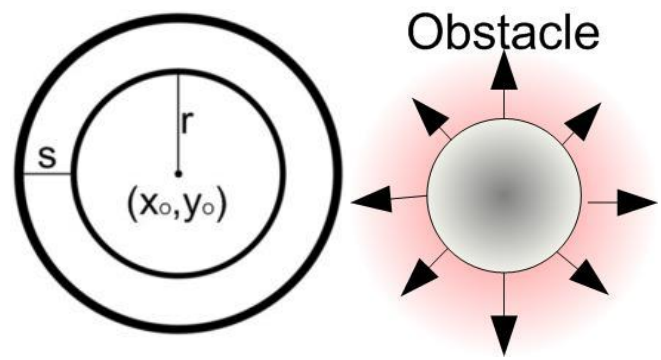

Figure 6

Action vectors of the repulsive potential field [11]

It is possible to calculate the repulsive action vector by applying a scalar potential field function to the robot's position and then calculating the gradient of that function.

$$
\nabla=[\nabla U(x, y)]=\left[\frac{\partial U}{\partial x}, \frac{\partial U}{\partial y}\right]
$$

Within the obstacle, the repulsive potential field is infinite and points out from the center of the obstacle. Beyond the circle of influence, the repulsive potential field is zero. The repulsive action vectorcan be calculated.

Having defined [14]

- $\left[x_{O}, y_{O}\right]$ as the obstacle position;

- $\quad r$ as the obstacle radius;

- $\left[x_{R}, y_{R}\right]$ as the robot position;

- $\quad s$ as the size of area of influence of the obstacle;

- $\beta$ as the strength of the repulsive field $(\beta>0)$

it is possible to compute $\nabla x$ and $\nabla y$ with the help of the steps given below:

1. Define the distance $d$ between the obstacle and the robot:

$$
d=\sqrt{\left(x_{O}-x_{R}\right)^{2}+\left(y_{R}-y_{O}\right)^{2}}
$$

2. Specify the angle $\theta$ between the robot and the obstacle:

$$
\theta=\tan ^{-1}\left(\frac{y_{O}-y_{R}}{x_{O}-x_{R}}\right)
$$

3. Set $\nabla x$ and $\nabla y$ based on the rules below: 
If $d<r$ then $\left\{\begin{array}{l}\nabla x=-\operatorname{sign}(\cos (\theta)) \infty \\ \nabla y=-\operatorname{sign}(\sin (\theta)) \infty\end{array}\right.$

If $r \leq d \leq s+r$ then $\left\{\begin{array}{l}\nabla x=-\beta(s+r-d) \cos (\theta) \\ \nabla y=-\beta(s+r-d) \sin (\theta)\end{array}\right.$

If $d>s+r$ then $\nabla x=\nabla y=0$

The attractive potential rules, similarly to the above-listed ones, are simple and describe three different robot behaviors with regards to its positionrelevant to the obstacle. It is essential to notice that all action vectors have to point away from the obstacle, hence the necessity to employ negative values [11].

- In the first rule (step 3), the robot is within the radius of the obstacle, so the action vector needs to be infinite, thus expressing the need to move away from the current location.

- In the second rule, when the robot is not inside the obstacle's radius but inside its area of influence, the action vector is assigned to a high value in order to express the need to move away from the current location.

- The third rule illustrates the case where the robot is outside the area of influence of the obstacle; the action vector is set to zero, and thus no repulsive forces are acting on the robot [14].

Special care must be taken when selecting the value of $s$, since the repulsive force only acts when the robot is inside the area of the obstacle's influence. If the value of $s$ is small, it may cause trajectory problems by abruptly changing its orientation in its path and leading to speed limitation of the robot. If $s$ has a high value, it can also result in problems in the robot's movement since it might limit its movement in tight spaces where the robot cannot pass.

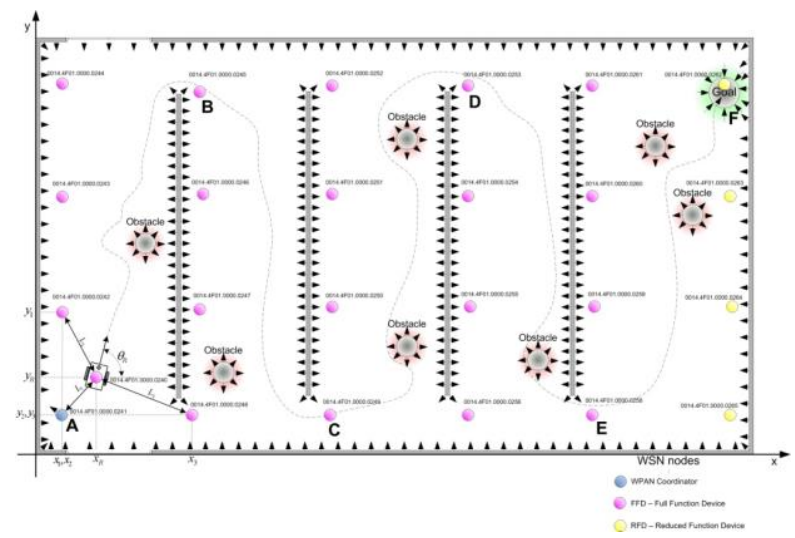

Figure 7

Potential Fields simulation 
The repulsive force has the objective of repelling the robot only if it is close to an obstacle and its velocity points towards that obstacle [11]. The robot is able to navigate along the map from point $\mathrm{A}$ to point $\mathrm{F}$ via points $\mathrm{B}, \mathrm{C}, \mathrm{D}, \mathrm{E}$ (see Fig. 7) collecting the environmental parameters.

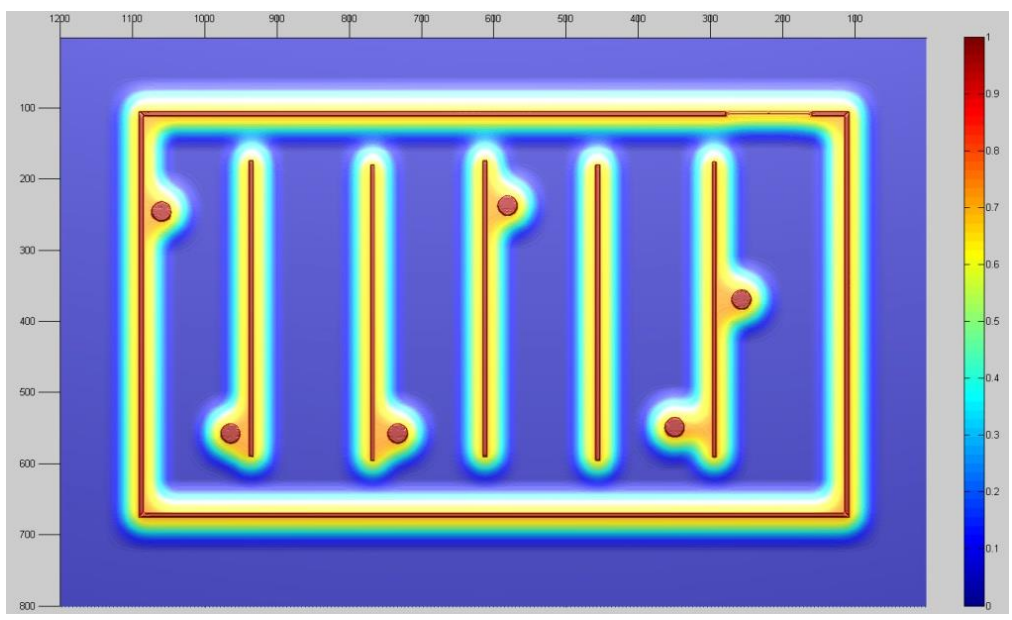

Figure 8

Top view of Potential Fields Values

The 3D surface presented inFig. 9 shows the force vector field of the repelling walls and obstacles, with further references indicated in [2,3]. The surface depicted inFig. 8 presents the repelling forces of the simulated environment. The map shown in Fig. 9 presents the absolute value of the forces of the simulation environment's potential fields.

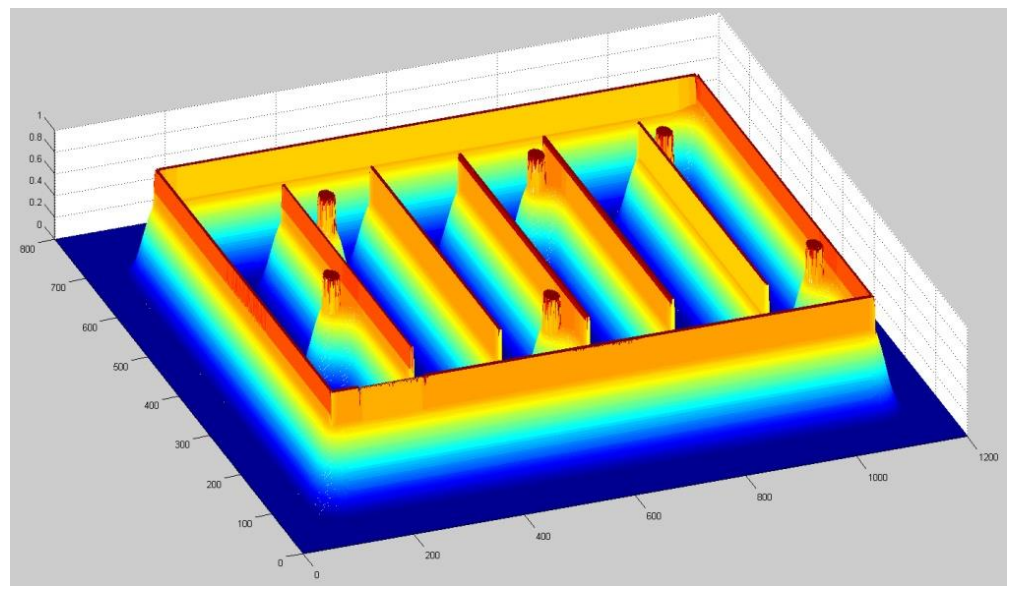

Figure 9

$3 \mathrm{D}$ view of potential field 
It is possible for the robot to be caught in local minima. This simply means that the robot has reached a location where the force is zero, with the opposing forces cancelling each other out. The result will be that the robot ceases to move and its final destination will never be reached [6].

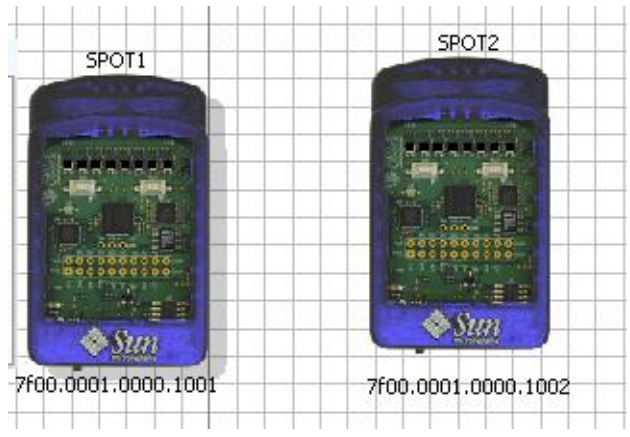

Figure 10

Java based Sun SPOT

The SunSPOT nodes (Fig. 10) radio communication is based on an embedded CC2420 radio circuit which is IEEE 802.15.4 compliant and works in the $2.4 \mathrm{GHz}$ to $2.4835 \mathrm{GHz}$ ISM bands [5]. The CC2420 datasheet specifies the equation to compute the received signal strength (in $\mathrm{dbm}$ ) using the raw values collected by the sensors.

\section{Conclusions}

In order to achieve efficient robot navigation in a controlled micro climatic environment the solution seems to be the application of the WSN as an aid for navigation. In this way we can ensure effective navigation by estimating the robot's position through the exact position of the mobile measuring stations, without the use offurther hardware. As a future extension of this experiment, this application can be implemented in the field of precision agriculture. Another area of possible improvement is the real-time tracking algorithm of the WSN aided navigation in order to increase the precision of the ranges not covered that lie beyond the reference nodes.

\section{References}

[1] Andrzej Pawlowski, Jose Luis Guzman, Francisco Rodríguez, Manuel Berenguel, José Sánchez, Sebastián Dormido, 2009, "Simulation Of Greenhouse Climate Monitoring And Control With Wireless Sensor Network And Event-Based Control” pp. 232-252

[2] C. H. Chiang, J. S. Liu and Y. S. Chou. 2009,“Comparing Path Length by Boundary Following Fast Matching Method and Bug Algorithms for Path Planning", Opportunities and Challenges for Next-Generation Artificial Intelligence, Springer, pp. 303-309 
[3] Gomide, R. L., Inamasu, R. Y., Queiroz, D. M., Mantovani, E. C., Santos, W. F., 2001,"An Automatic Data Acquisition and Control Mobile Laboratory Network for Crop Production Systems Data Management and Spatial Variability Studies in the Brazilian Center-West Region", ASAE Paper No.: 01-1046. The American Society of Agriculture Engineers, St. Joseph, Michigan, USA

[4] Gy. Mester, 2009, „Wireless Sensor-based Control of Mobile Robot Motion“, Proceeding of the IEEE SISY 2009, pp 81-84, Subotica, Serbia

[5] István Matijevics and Janos Simon 2010, "Improving Greenhouse's Automation and Data Acquisition with Mobile Robot Controlled System via Wireless Sensor Network", Wireless Sensor Networks: ApplicationCentric Design, Geoff V Merrett and Yen Kheng Tan (Ed.), InTech

[6] J. Simon, G. Martinović, 2009, "Web Based Distant Monitoring and Control for Greenhouse Systems Using the Sun SPOT Modules", Proceedings of the Conference SISY 2009, pp. 1-5, Subotica, Serbia

[7] J. Vasu, L. Shahram, 2008, "Comprehensive Study of Routing Management in Wireless Sensor Networks- Part-1"

[8] K. Kreichbaum. 2006, Tools and Algorithms for Mobile Robot Navigation with Uncertain Localization. $\mathrm{PhD}$ thesis, California Institute of Technology

[9] L. Gonda, C. Cugnasca, 2006, “A Proposal of Greenhouse Control Using Wireless Sensor Networks" In Proceedings of 4thWorld Congress Conference on Computers in Agriculture and Natural Resources, Orlando, Florida, USA

[10] Liu, G., Ying, Y., 2003, "Application of Bluetooth Technology in Greenhouse Environment, Monitor and Control”, J. Zhejiang Univ., Agric. Life Sci. 29, 329-334

[11] Luca Bencini, Davide Di Palma, Giovanni Collodi, Antonio Manes and Gianfranco Manes, 2010,"Wireless Sensor Networks for On-Field Agricultural Management Process", Wireless Sensor Networks: Application-Centric Design, Geoff V Merrett and Yen Kheng Tan (Ed.), InTech

[12] M. J. Matarić, 2007, The Robotics Primer. The MIT Press, $1^{\text {st }}$ edition

[13] Mizunuma, M., Katoh, T., Hata, S., 2003, “Applying IT to farm fields”, A Wireless LAN. NTT Tech. Rev. 1, pp. 56-60

[14] O. Khatib, 1985, "The Potential Field Approach and Operational Space Formulation in Robot Control" Proc. Fourth Yale Workshop on Applications of Adaptive Systems Theory, Yale University, New Haven, Connecticut, pp. 208-214

[15] O. Khatib, 1986, "Real-Time Obstacle Avoidance for Manipulators and Mobile Robots", Int. J. of Robotic Research, Vol. 5, No. 1, p. 60 
[16] R. Langer, L. Coelho and G. Oliveira. 2007, "K-Bug, a New Bug Approach for Mobile Robot's Path Planning", IEEE International Conference on Control Applications, pp. 403-408

[17] Roland Siegwart and Illah R., 2004, "Introduction to Autonomous Mobile Robots", Nourbakhsh

[18] Serodio, C., Cunha, J.B., Morais, R., Couto, C. A., Monteiro, J. L., 2001, "A Networked Platform for Agricultural Management Systems", In: Computers and Electronics in Agriculture, vol. 31. Elsevier, pp. 75-90

[19] V. Lumelsky and A. Stepanov. 1987, "Path Planning Strategies for a Point Mobile Automaton Moving Amidst Unknown Obstacles of Arbitrary Shape", Algorithmica, Vol. 2, pp. 403-430

[20] X. Feng, T. Yu-Chu, S. Yanjun, S. Youxian, 2007, "Wireless Sensor/Actuator Network Design for Mobile Control Applications. Sensors" Proceedings of the Conference

[21] Y. Takahashi, T. Komeda, H. Koyama. 2004, "Development of Assistive Mobile Robot System”, Amos. Advanced Robotics, Vol. 18, No. 5

[22] Silvester Pletl, Péter Gál, DraganKukolj, László Gogolák, 2010, “An Optimizing Coverage in Mobile Wireless Sensor Networks", $8^{\text {th }}$ International Symposium on Intelligent Systems and Informatics - SISY 2010, Subotica

[23] Joao Sena Esteves, Adriano Carvalho and Carlos Couto, 2003, "Generalized Geometric Triangulation Algorithm for Mobile Robot Absolute Self-Localization", ISIE 2003 - 2003 IEEE International Symposium on Industrial Electronics, Rio de Janeiro, Brazil, pp 1-12 